\title{
Short - term Forecast Model of Sugar Futures Price Based on Seasonal Decomposition
}

\author{
Shuang Cheng \\ School of Finance, Guizhou University of Finance and Economics, Guiyang Guizhou \\ 550025, China
}

\begin{abstract}
With the sugar futures price forecast for the theme in this article, from the seasonal character-istics of white sugar, in January 2006 - June 2017 the sugar futures first month closing price as the foundation of data, using the seasonal decomposition method to the sugar futures price forecast of July to September, and the predicted value and actual value were analyzed. The seasonal factor of the original time series was precipitated by seasonal decomposition method and the seasonal adjustment sequence was obtained. Then the seasonal adjustment sequence is predicted by exponential smoothing. Finally, the seasonal factors are replenished to the trend forecast, and the price prediction results of the sugar futures are obtained. The research results show that the price of the sugar futures fluctuates in fluctuation, and the author puts forward four related policy suggestions.
\end{abstract}

\section{Keywords}

The sugar futures; Price prediction; Seasonal decomposition method; Exponential smoothing method

\section{基于季节分解法的白糖期货价格短期预测模型}

\author{
程爽 \\ 贵州财经大学金融学院, 贵阳 550025, 中国
}

摘要：本文以白糖期货价格预测为主题，从白糖的季节性特征入手，以 2006 年 1 月-2017 年 6 月的白糖期货主力合约的月收盘价为数据基础，运用季节分解法对 7 到 9 月的白糖期货 价格进行预测, 并将预测值与实际值进行对比分析。首先用季节分解法将原始时间序列中的 季节因子析出, 并得到季节调整序列; 然后采用指数平滑法对季节调整序列进行趋势预测; 最后将季节因子回补到趋势预测值当中，得出白糖期货价格预测结果。研究结果发现白糖期 货价格呈现上下震荡波动的趋势，最后笔者提出四点相关的政策建议。

关键词：白糖期货；价格预测；季节分解法；指数平滑法 


\section{1. 引言}

我国的食糖产量位居世界前列, 目前已成为全球范围内食糖最主要的 生产和消费地区之一。由于经济社会 发展和生活水平与质量不断提高, 使 得食糖的消费水平不断提高。截止到 17 年上半年榨季生产销售相关数据显 示, 全国范围内食糖的销售数量总计 达到 603.16 万吨, 累计销糖率已达到 $64.94 \%$, 高于上榨季同期的 $61.9 \%$ （数据来源：新浪财经-期货网站）。

上世纪七八十年代以来, 我国政 府逐渐放宽了白糖的定价, 但是由于 白糖市场缺乏具有权威性的价格引 导，国内白糖市场价格频繁发生较大 的起伏。为合理有效地解决价格的波 动问题, 白糖期货在 2006 年 1 月与郑 州上市, 自此, 郑州商品交易所白糖 的期货贸易长期保持较为火热的状 态，随后几年我国的白糖期货贸易量 也呈现出迅速上升的趋势, 由此也促 进了我国白糖市场价格的制定。

本文在借鉴前人研究成果的基础 之上, 提出运用季节分解的理论和方 法对白糖期货价格进行短期预测和分 析, 以此可以较好地规避季节性时间 序列的不稳定性在一般的分析和预测 过程中所产生的不利影响, 为日后关 于这一问题的研究提供了新的方向。

国外关于期货价格预测方面的研 究, 主要是基于市场具有有效性的相 关理论。Yang J 等人（2001）指出相 关资产的拒藏难易程度会影响到期货 价格预测的准确性。目前国外普遍使 用 ARIMA 模型来研究价格预测问题, 该模型最早由 Box 和 Jenkins (1976) 创造, 适用于对期货价格进行短期预 测分析。随后, Barkoulas 等 (1997) 在此基础上提出 ARFIMA 模型分析处 于不断变化过程中的期货价格预测问 题。而随后 Benz E 和 Daskalakis $\mathrm{G}$ 等 人（2009）针对碳排放的现货与期货
构建预测模型，这也为日后有关期货 价格的研究发挥了重要的指导作用。

通过对上述国外文献的整理，可 以看出国外专家学者对于这一问题的 研究都是立足于微观层面, 偏好对个 人投资进行分析的基础上的探索，而 我国人口众多、幅员辽阔, 对于农产 品的需求量巨大, 因此对于我国这一 问题应当立足于宏观角度而非仅仅基 于微观角度展开相关的研究与探索。

相较于国外来说，国内期货市场 起步较晚, 对于期货市场的研究都是 在借鉴国外较为成熟发达的成果的基 础上展开的。其中, 褚冬 (2015) 利 用 GARCH 模型对大豆期货价格进行 短期预测。李战江等人 (2012) 和费 婧文 (2017) 运用 ARIMA 模型 分别 对沪深 300 股指期货和黄金期货结算 价格在短期内的变动趋势进行预测和 分析。另外, 王海军等人 (2009) 收 集整理关于强筋小麦的期货数据, 采 用神经网络预测方法对于小麦期货价 格进行预测。张凯（2012）提出基于 非线性组合来构建相关模型对世界原 油期货价格进行预测分析。许贵阳

（2014）运用灰色预测方法预测黄金 期货价格, 结果表明所构建模型对其 价格变动趋势能有效预测, 但是模型 具有一定的适用条件。

综合上述文献综述, 我们可以看 出, 现阶段国内对于期货价格预测等 方面的研究仍是在建立相关模型的基 础上, 其存在着变量选取考虑因素不 够全面以及预测模型选择局限使得分 析不具有普遍性等问题, 而且所采用 的研究方法和思路具有一定的单一 性, 对于问题的分析往往只关注到表 面现象, 没有深虑到白糖的季节性变 动等更加深层次的内容。因此, 针对 国内对该问题研究的不足, 本文提出 具有一定创新性的思路, 立足于白糖 季节性变动等相关特征, 通过采用季 
节分解相关方法, 对这一问题进行较 为深入地实证研究与探索。

\section{2. 我国白糖期货市场的现状分析}

\section{1 市场现状}

06 年之后, 白糖期货市场逐渐成 为社会关注的重点, 越来越多的投资 人和专家学者开始探索和分析这一市 场。随后的十几年, 其贸易总量以及 平均每天贸易量和每天的平均持仓额 都有飞速上涨。根据相关统计资料显 示，到目前为止我国白糖的期货市场 总体良好, 期货品种较为丰富, 在世 界范围内, 其贸易量在农产品衍生物 的市场中一直位居前列。

\section{2 影响白糖期货价格的因素}

\subsection{1 白糖现货市场的供给和需求}

就供给方面而言, 通过查阅美国 农业部的相关统计资料，我们发现到 目前为止世界上最大的食用糖的产区 主要是巴西、中国、印度和欧盟以及 泰国, 这些地区的总产量已经超过全 球总产量的一半, 在这些国家和地区 糖类市场的发展在其整个经济的发展 进步中发挥着巨大的作用。

根据需求情况来看, 世界上食用 糖最大的消费区是印度、中国、巴西 以及欧盟与美国, 这些国家或者地区 的消费量总额在全球消费总额中所占 比重已经超过了 $50 \%$, 我国食用糖消 费量在世界排名中也靠前, 平均每年 的消费量长期保持在大约 1500 万吨。

\subsection{2 气候因素}

甘蔗和甜菜作为生产加工糖类的 主要原材料, 由于属于农产品, 气候 相关因素势必会对其产生作用。其中 甘蔗种植就比较适合在热带或者温带 地区, 该农作物的种植都需要温度以 及湿度、光照度达到一定的要求, 过 于干燥以及寒冷都会影响其生长, 也
就是说遇到干旱或者暴雪等自然灾害 都会对其种植产生不利影响。

\subsection{3 季节性}

糖类的销售具有明显的季节性特 征, 具体来说就是春节前后和夏季由 于冷饮的需求量巨大以及中秋月饼等 糕点的制作量巨大等等, 这些时期都 是糖类销售的高峰期; 剩下的时期, 销售量明显较低，其中，一般将每年 的 10 月至第二年 4 月称为压榨季; 第 二年 5 月至 9 月称为纯销售季即非椥 季。正是由于这种季节性变动, 使得 其价格也呈现出一定的起伏。由此我 们可以看出白糖所具有的季节性特征 会对其价格产生很大影响。

\subsection{4 替代品的价格等情况}

替代品就是取代糖类发挥甜味调 节的相关产品，在我们的日常生活中 可以发现许多能够起到甜味调节的物 质, 随着社会进步, 甜味剂的出现打 破了原本白糖市场的供求局面, 它的 出现与原本的糖类抢占市场, 从而对 原本糖类的价格产生一定的作用。常 用的甜味剂主要包括淀粉糖、麦芽糖 浆、葡萄糖以及果葡糖浆等等。

\section{3. 实证分析}

\section{1 方法与模型的选择}

季节性变量通常反映的是在一年 或者更短的时期内（比如天、星期、 月或季度等) 的变动情况, 这段时期 内在诸如气候、经济、政治以及社会 风俗等众多因素的作用下所表现出的 相应的高峰值或者低谷值。具有波动 性的季节性时间序列正是由于其不稳 定性使得其变动情况相较于其他时间 序列来说复杂程度更高, 从而在一定 程度上加大了预测和分析的难度。另 外, 在对时间序列的相关数据进行分 析和建模的过程中, 季节分解也扮演 
着重要的角色。时间序列的成分包 括：趋势、季节变动、循环波动、不 规则变动, 季节分解法的基本思想是 将季节变化从原时间序列的变异中去 除, 并生成由这 4 部分构成的序列来 满足进一步的分析要求。（1）加法模 型：加法模型假设原序列的信息可被 分解为 4 个相加部分序列总变异=趋势

$(\mathrm{T})+$ 季节变动 $(\mathrm{S})+$ 循环波动 $(\mathrm{C})$ +不规则波动（I）。（2）乘法模型: 乘法模型假设原序列的信息可被分解 为 4 个相乘部分 序列总变异 $=$ 趋势

( $\mathrm{T})$ *季节变动（ $\mathrm{S}$ ） *循环波动 $(\mathrm{C})$ *不规则波动（I）。去除明显的季节波 动, 然后建立回归模型, 从而能够使 预测的准确度和精确度得到大大的增 强。所以, 运用这种方法可以很好地 对具有不稳定性的季节性时间序列进 行分析和预测。基于以上原因，本文 提出一种基于季节分解法的白糖期货 价格短期预测模型。

\section{2 数据来源以及选取}

由于统计资料中关于期货价格的 数据有很多, 因此如何对相关数据做 出合理有效地选择, 确定具有代表性 的数据, 都需要深思熟虑。一般而言 期货的开盘价以及最高和最低价格具 有一定的脆弱性, 很容易受到不确定 因素的干扰，所以这些价格信息不具 有代表性，但是当日的收盘价格作为 该日最后的成交价格，其所反映的结 果也是该交易日相关信息影响价格的 最好体现, 所以国内外专家学者对这 方面问题的研究大多数确定的具有代 表性的价格都是每日的收盘价格。本 文的研究对象是郑商所的白糖期货, 所使用的数据主要来源于郑商所官网 以及东方财富网的每月收盘价格, 时 间跨度为 2006 年 1 月至 2017 年 6 月。

因为白糖期货价格的变动趋势在 总体上呈现不稳定性, 同时具有季节 变动性等特征, 其所涉及的时间序列
趋势具有二重性, 本文运用季节分解 法建立白糖期货价格短期预测模型。

基本步骤包括：（1）季节分解：运用 季节分解法析出原始时间序列中的季 节因子，并得到季节调整序列

（SAS）。（2）趋势预测：运用指数 平滑法在上一步所得的结果的基础之 上进行趋势预测, 得出预测周期内的 趋势预测值。（3）价格预测：根据乘 法模型将季节因子回补到趋势预测值 中, 得到预测的价格, 即得出 7-9 月的 预测值及上下置信区间。

\section{3 实证结果与分析}

第一步: 确定并分离季节成分。 运用SPSS24.0软件提供的季节分解功 能, 选择乘法模型, 得出季节分解 表, 如表1所示。显然有: 原始序列= 季节因子 $\times$ 季节性调整序列 $\times$ 平滑趋 势周期序列 $\times$ 不规则 (误差) 因子。 通过序时图可以看出季节调整序列

（SAS）走势，运用序时图功能可得到 以下图形。从图中可以看出白糖期货 价格呈现出上下震荡的趋势特征。

\section{表1 白糖期货价格季节分解表}

周期性分解

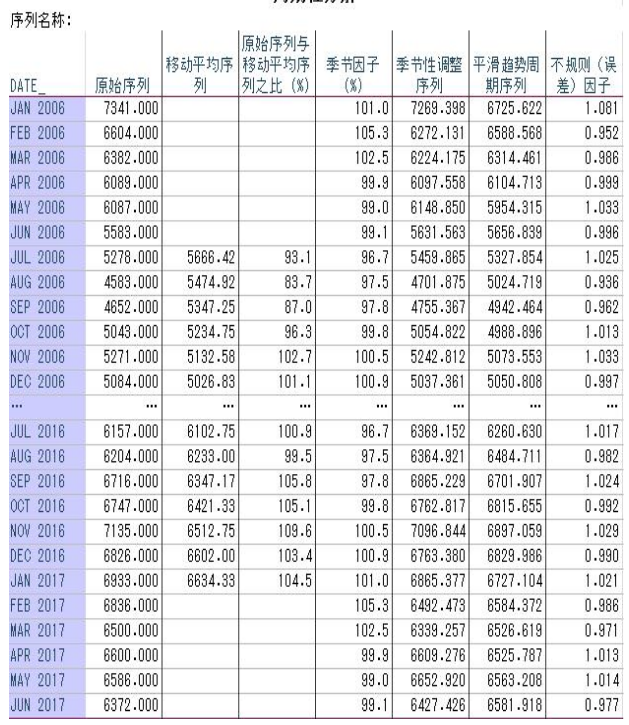




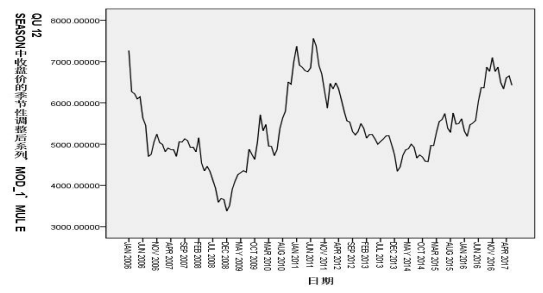

图1 季节调整后白糖期货价格

第二步：运用指数平滑法对收盘 价格进行预测。预测周期设定为 2017 年7到9月, 并且还给出了预测值的上 下置信区间, 具体数值如表 2 所示。

表 2 预测结果

\begin{tabular}{|c|c|c|c|c|}
\hline \multicolumn{2}{|l|}{ 预测模型 } & 2017.07 & 2017.08 & 2017.09 \\
\hline $\begin{array}{l}\text { SEASON 中 } \\
\text { 收盘价的 } \\
\text { 季节性调 }\end{array}$ & 预测 & 6416.86080 & 6355.40800 & 6377.63815 \\
\hline $\begin{array}{l}\text { 整后系列, } \\
\text { MOD_1 , }\end{array}$ & UCL & 6977. 69743 & 7148.15512 & 7348. 38954 \\
\hline $\begin{array}{l}\text { MUL EQU } \\
\text { 12-模型_1 }\end{array}$ & LCL & 5856.02418 & 5562.66088 & 5406. 88675 \\
\hline
\end{tabular}

注: 对于每个模型, 预测从所请求估算期范围内的最后一个非缺 失值之后开始, 并结束于最后一个所有预测变量都有可用的非缺失值 的周期, 或者在所请求预测期的结束日期结束, 以较早者为准。

第三步，季节因子回补。即用季 节因子乘以季节调整后序列得到预测 值。由于期货价格数值均为整数, 故 忽略小数点。同理, 运用指数平滑法 对原始序列进行预测, 得到未经季节 调整的期货价格预测值, 将得到的这 两种预测值放于同一表中并计算误 差, 得到表 3 , 经过对比发现经过季节 因子回补的预测值能更好的预测期货 价格，故季节分解模型能够较好的预 测白糖期货价格，对白糖期货的季节 性进行简要分析。另一方面, 模型还 给出价格预测值上下置信区间, 为价 格预测值的调整提供一定的空间。

\begin{tabular}{c|l|l|l|l|l}
\multicolumn{7}{c}{ 表 3 预测结果对比 } \\
\hline 月份 & 实际值 & $\begin{array}{l}\text { 回补季节 } \\
\text { 因子后的 } \\
\text { 预测值 }\end{array}$ & 误差 & $\begin{array}{l}\text { 原始序列 } \\
\text { 预测值 }\end{array}$ & 误差 \\
\hline 2017.7 & 6128 & 6205 & $1.26 \%$ & 6229 & $1.64 \%$ \\
2017.8 & 6413 & 6197 & $-3.37 \%$ & 6212 & $-3.1 \%$ \\
2017.9 & 6117 & 6237 & $1.96 \%$ & 6253 & $2.22 \%$ \\
\hline 注: 误差= (预测值一实际值) /实际值
\end{tabular}

在经过不断进步和完善之后, 白 糖期货在我国已经发展成期货市场中
较为完善的交易品种, 其成熟度也有 明显的提高, 因此价格方面呈现复杂 性和多样性, 这中间既有外部因素又 有内部因素影响。正是由于白糖期货 价格的变动趋势在总体上呈现一定的 不稳定性, 同时具有季节变动性等特 征, 其所涉及的时间序列趋势具有二 重性, 因此利用季节分解法从原有的 时间序列中析出季节因子，并在随后 运用指数平滑法对所得出的季节调整 序列的趋势进行相关分析和预测，回 补预测值从而在最后达到对白糖期货 价格预测的目的, 通过实证研究也检 验了该方法是可行的, 所得出的结果 也是合理有效的, 同时从某种程度 上，该方法的使用也为日后研究白糖 期货价格等相关问题指明了新的方向 以及提供了新的理论依据, 具有一定 的指导作用。从模型分析的结果可以 看出, 季节调整后白糖期货价格呈现 上下震荡的趋势，振幅大概在 3500 6500 之间, 震荡周期从峰顶到峰谷再 到峰顶大概是 5 年左右，因而白糖期 货价格变动是一个长期的趋势。

郑商所白糖期货上市对于我国食 糖业的发展具有重要战略意义, 但我 国期货市场功能仍然不够完善，应积 极规范我国食糖市场价格形成机制, 努力形成以期货价格为主要参考的多 层次和高效率的食糖市场价格体系。

\section{4. 政策启示}

（1）加强调控持续市场风险准备

我国的期货市场历史不长，还有 很多需要完善的地方, 鉴于白糖期货 价格上下震荡不稳定的特征, 一旦在 短期内发现白糖的期货价格出现明显 的起伏时, 也就表明未来一段时期内 这种起伏不会迅速消除, 这种不稳定 的状态将会维持下去。所以，相关投 资人员在发现出现这种不稳定的起伏 状况时要迅速及时地采取相应措施防 范和规避可能存在的风险, 降低其所 
参与的市场活动的不稳定性, 同时相 关政府工作人员以及监督管理人员也 要制定相关政策和措施对整个市场进 行调控, 从而使市场逐步趋于稳定。

（2）鼓励研究白糖期货市场, 并 强化对该市场贸易情况的监督、管理 与控制

通常来说，相关期货市场的起伏 情况可以在某种程度上通过该市场相 关期货贸易成交量的数值变动来体 现，因此我们应该不断探索这些期货 的贸易成交量所能够显示的更深层次 的信息，进而可以从另一方面对相关 市场的起伏情况进行分析研究, 从而 其在某种程度上能够对市场监督管理 者起到一定的警报作用，从而提醒市 场监督管理者需要对贸易成交量进行 必要的观测以及监督和管理。

（3）适度增加白糖现货储备

总体而言甜菜生产水平和效率并 不高, 政府部门对其扶持程度也偏 低, 而且没有形成较为成熟的产业链 与更加高效的生产模式, 这些因素限 制了甜菜数量的上升。所以当白糖期 货市场风险呈现上升趋势时，可以通 过运用诸如对相关粮食作物制定合理 的最低收购价以及临时收储政策等措 施和方法来加大对白糖现货的持有。

（4）进一步加强期货交割的建设

众所周知, 交割在期货市场的贸 易活动中是不可或缺, 它既是连接期 货和现货两大市场的桥梁，同时也能 够更好地保证期货市场相关作用得到 有效地发挥。因此, 郑商所在全国范 围内关于强化白糖期货交割仓库的建 设以及对白糖入库制定严格、准确、 合理的标准等相关工作势在必行; 另 外, 还需要提高交割方式的灵活性, 立足于现行的集中交割以及期转现等 方式, 努力创新和提倡多元化的方 式, 同时还可以适当简化相关业务手 续和程序, 从而可以加快流动速度。

\section{参考文献}

[1]Yang, J. Bessler D A, Leatham, D.J. Asset storability and price discovery in commodity futures markets: a new book[J]. Journal of Futures Markets, 2001, 21(3): 279-300.

[2]Box, and Jenkins.Time Series Analysis: An Applied Approach [M].1976.

[3]Barkoulas et al. Fractional Dynamics in International Commodity Prices $[\mathrm{J}]$. J ournal of Futures Markets, 1997, (17): 161-189.

[4]Benze,Trucks. Modeling the price dynamics of $\mathrm{CO}_{2}$ emission allowances[J]. Energy Econ, 2009, 31(1): 4-15.

[5]褚冬. 时间序列模型对大豆期货价格 的预测比较研究 [J]. 金融经济, 2015, (24): 116-119.

[6]李战江等.基于 ARIMA 模型的沪深 300 股指期货价格预测研究 [J]. 鲁东 大学学报, 2013, (01): 22-24.

[7]费婧文. 基于 ARIMA 模型对我国黄 金期货价格分析与预测 $[\mathrm{J}]$. 当代经济, 2017, (09): 148-150

[8]王海军,白玟,贾兆立等. 基于粒子群 神经网络的期货价格预测 $[\mathrm{J}]$. 计算机 工程与设计，2009，(10): 2428$2430+2434$.

[9]张凯,沙峰. 基于非线性组合方法的 石油期货价格预测研究 [J]. 计算机仿 真, 2012,(07): 379-382.

[10]许贵阳. 基于灰色预测方法的中国 黄金期货价格预测模型 [J]. 黄金, 2014, (01): 8-11.

[11]张红星. 郑州白糖期货市场功能实 证研究 $[\mathrm{J}]$. 金融理论与实践, 2010, (10): 90-93.

[12]张红梅, 孔容. 基于季节分解法的卷 烟销售短期预测模型 $[\mathrm{J}]$. 现代物业, 2012, (11): 86-89. 